\title{
II = Paris, Bibliothèque nationale de France, gr. 2821
}

\author{
I. Costantinopoli, XIV sec.; II. [Restauro] XV sec.
}

Comp.; ff. A, 1-103, A'; num. nel marg. sup. est. a penna; sul f. Ar è apposto un foglio con il pinax del codice, di mano di Matteo Devaris (Smith 1975, 96-97 n. 77), e con una nota in latino, Aristophanis ms p(ri)m(a)e comedi(a)e cum glosis e l'indicazione $n^{\circ} 8$; sul f. 1r sono riportate le segnature precedenti $(1531,3328)$. Legatura in cuoio scuro con ornamentazione formata da tre bordure rettangolari concentriche sul piatto anteriore e posteriore, in cui sono impressi disegni a ferri rettangolari rappresentanti una treccia e dei fiori; sulla parte inferiore del dorso, da cui sporgono quattro nervature, è applicata un'etichetta con la segnatura attuale.

Il codice è formato da due unità: la più antica risale alla metà del XIV secolo ed è stata realizzata a Costantinopoli; quella più recente è un restauro riferibile al XV secolo, atto a restituire un bifoglio della prima unità.

\section{I. ff. 1-11, 14-103 (Costantinopoli, XIV sec.)}

\begin{abstract}
Cart.; fil. ff. 2, 3, 6, 7, 10, 15, 18, 19, 22, 23, 26, 28, 29, 31, 34, 36, 7, 39, 42, 44, 45, 47, 51, 53, 54, 56, 60, $62,63,64,65,67,70,72,73,75,78,80,81,83,86,87,90,91,93,96,100,102$ simile a cercles $(\mathrm{M} / \mathrm{Tr}$ nr. 2161,1360$) ; 1-6^{8}(48), 7-8^{10}(68), 9-12^{8}(102), 13^{2+1}(104)$; num. fasc. doppia, una più antica presente nel marg. sup. est. del recto del primo foglio dei fascicoli, procede dal terzo fino all'ultimo, numerati da y' (17) a ıy' (101); una più recente presente nel marg. inf. est. del verso dell'ultimo

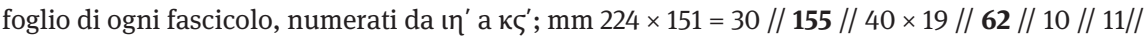
30 // 19; una colonna, 11. 10-11. 20, unità di rigatura mm 8; rigatura tracciata a secco sul recto di ogni foglio, tipo S/L 30D1 (Muz 2-21/0/0/J)(f. 44r); mm $225 \times 147=18$ // 180 // $27 \times 18$ // 109 // 4 // 17; a piena pagina, 1l. 37-1l. 41, unità di rigatura $\mathrm{mm} 4$; rigatura tracciata a secco sul recto dei fogli, tipo S/L 10D1n (Muz 1-2/0/0/J) (f. 2r).
\end{abstract}

\section{Scrittura}

Nell'unità è presente un'unica mano: <Isacco Argiro (Bianconi 2010, 493 e Mondrain 2007 [2008], 169)> (ff. 1r-11v, 14r-103v), che ha adoperato un doppio registro grafico: uno corsivo per le sezioni in prosa (ff. 1r-2r, 33r, 68rv, 69r) e gli scholia a margine delle sezioni poetiche (ff. $2 \mathrm{v}-11 \mathrm{v}, 14 \mathrm{r}-32 \mathrm{v}, 33 \mathrm{v}-67 \mathrm{v}, 69 \mathrm{v}-103 \mathrm{v}$ ); uno posato per la stesura dei testi poetici veri e propri (ff. 2v-11v, 14r-32v, 33v-67v, 69v-103v). 


\section{Decorazione}

Ad Argiro si deve anche la rubricatio.

\section{Interventi successivi}

Oltre alla mano di Argiro, cui si deve l'apparato critico-esegetico che incornicia il testo principale, è possibile distinguere in alcuni fogli una seconda mano, posteriore di almeno un secolo, che appone annotazioni o segnalazioni (ff. 34v, 39v, 56rv, 63v, 73r, 74v, 77r, 78rv, 79r, 80v, 81rv, 84v, 90v), spesso precedute da una manicula.

\section{Contenuto}

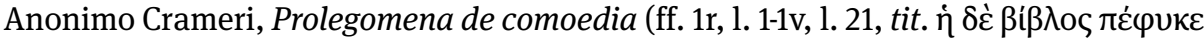

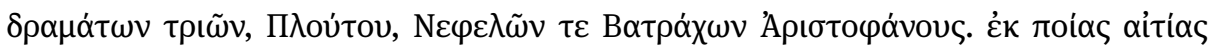

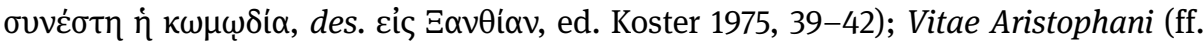

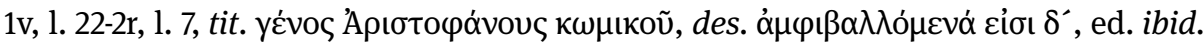

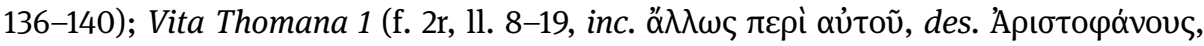
ed. ibid. 146-147); Argumentum Plutum (f. 2r, 11. 20-34, inc. ن்

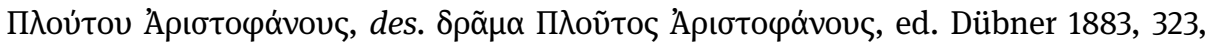

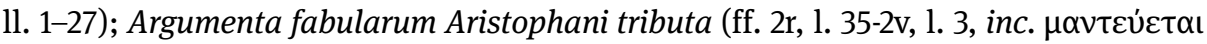

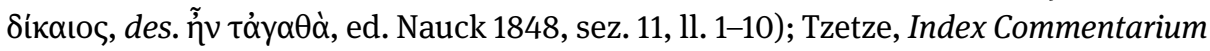

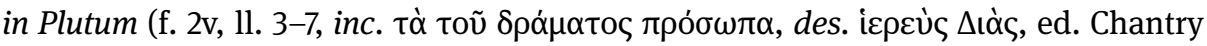

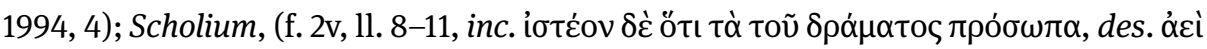

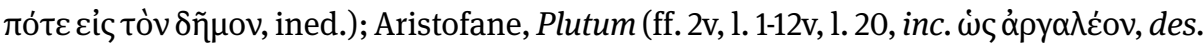

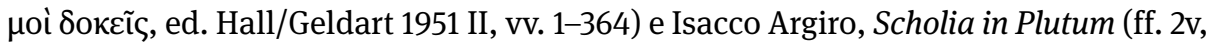

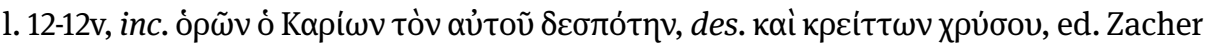

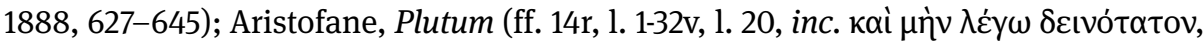

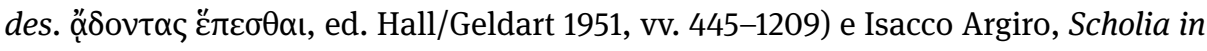

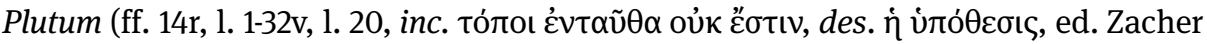

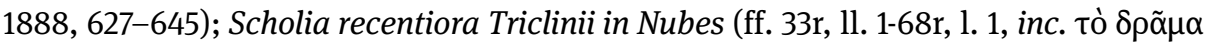

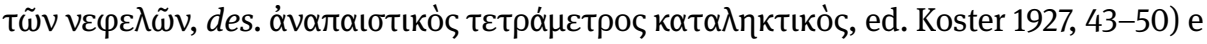

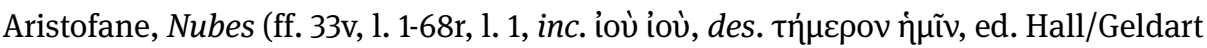
1951 I, vv. 1-1511); Isacco Argiro, De metris poeticis (ff. 68r, 1. 3-68v, 1. 15, tit. $\mu \varepsilon ́ \tau \rho \alpha ~ \tau \imath v \tilde{\omega} v$

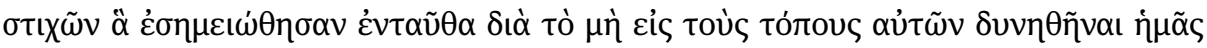

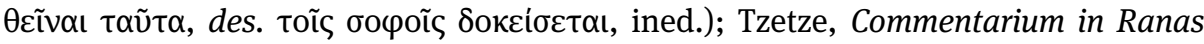

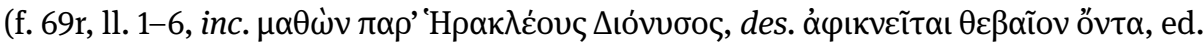

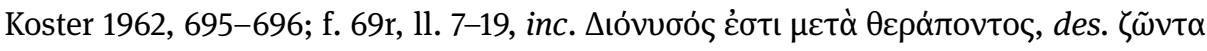

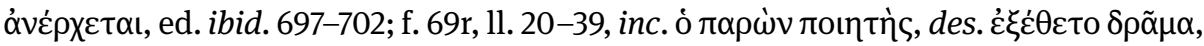

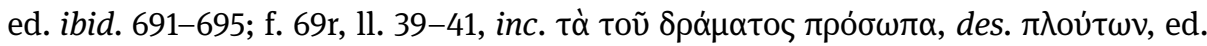




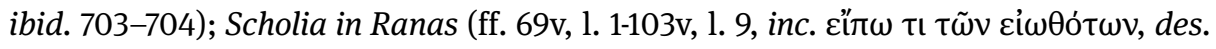

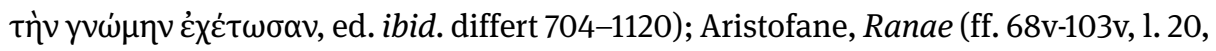

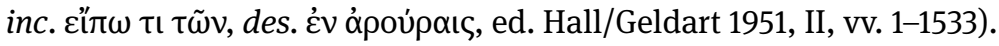

\section{II. ff. 12-13 (XV sec.)}

Cart.; fil. assente; $14^{2}$ (13); mm $125 \times 148=25 / / 150 / / 25 \times 12$ // $\mathbf{6 0} / / 71$; una colonna, ll. 20, unità di rigatura $\mathrm{mm}$ 7; rigatura tracciata a secco sul recto dei fogli, tipo S/L 00D1 (Muz 1-1/0/0/J) (f. 13).

\section{Scrittura}

Questi fogli sono stati vergati da un solo scriba: mano B (ff. 12r, 1. 1-13v, 1. 20), scrittura riferibile al XV secolo.

\section{Decorazione}

Sono vergati in inchiostro rosso, sbiadito, i nomi dei personaggi al principio di ogni battuta.

\section{Contenuto}

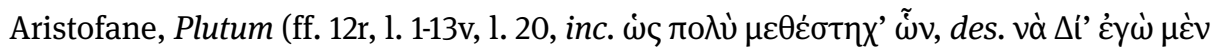
oov̉, ed. Hall/Geldart 1951, vv. 365-444) e Isacco Argiro, Scholia in Plutum (ff. 12r-13v,

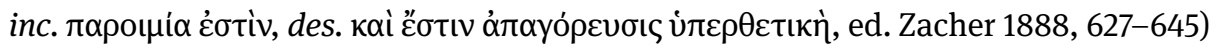

\section{Relazioni stemmatiche}

Ole Langwitz Smith, che per primo notò l'unità del Parm. 154 e del Par. gr. 2821 (Smith 1974, 414-415) - cui successivamente è stato aggiunto anche il Par. gr. 2758 (Mondrain 2005, 54) -, aveva attribuito le recensioni e i corredi scoliastici recati nei due esemplari all'attività giovanile di Demetrio Triclinio (Smith 1975, 95). Willem J. W. Koster e Charles N. Eberline hanno escluso l'autorialità tricliniana, considerando la recensione del commediografo, e di conseguenza anche quella euripidea, come post-tricliniane (Koster 1975, 50-53; Eberline 1980, 24-25). Questa ipotesi ha trovato conferma nell'identificazione del copista dei manoscritti con Argiro (Mondrain 2007 [2008], 169-170, Bianconi 2010, 493). Secondo Smith, il restauro dei ff. 12-13 del Par. gr. 2821 sarebbe avvenuto quando il codice era già passato nella biblioteca del cardinale Ridolfi. Poli- 
ziano si servì di questo manoscritto per ricavare alcuni degli scolii ad Aristofane da lui apposti nel Par. gr. 3069 (Pontani 2002, 140).

\section{Bibliografia}

\section{Cataloghi}

Omont (1888) 45.

\section{Edizioni}

Chantry (1994) IV; Dünber (1883); Koster (1927); Koster (1962); Koster (1975); Hall/Geldart (1951); Nauck (1848); Zacher (1888).

\section{Codicologia e paleografia}

Bianconi (2010) 492-493; Eberline (1980) 24-25, 123; Eleuteri (1993) 39-41; Koster (1957) 37, 50-53; Mondrain (2007) [2008] 169-170; Mondrain (2006-2007, 2008) 52-54; Muratore (2009) I, 182, 282 n. 49; II, 120, 122, 156, 411, 454, 508, 537, 574, 702, 804; Pontani (2002) 140; Smith (1974) 414-415; Smith (1975) 49, 82, 91, 95-112; Smith (1981-1982) 239-262; Smith (1992) 187-229. 